\title{
ADAMANDIOS KORAIIS, ÚLTIMO REPRESENTANTE DE LA ILUSTRACIÓN GRIEGA
}

\author{
Miguel Castillo Didier \\ Universidad de Chile, Chile
}

Resumen: Entre las grandes figuras de la Ilustración griega, se distingue la de Adamandios Koraís. Nacido y formado inicialmente en Esmirna, estudia medicina en Montpellier, adentrándose al mismo tiempo en la filosofía clásica. Se establece en París en 1788, donde vive hasta su muerte. Desarrolla una intensa actividad pedagógica y editora de textos políticos patrióticos y pedagógicos y de una vasta Biblioteca Clásica Griega. Si bien apoyó firmemente la Revolución de la Independencia de Grecia (1821-1830), criticó las divisiones entre los patriotas. Criticó también la obra de Ioanis Kapodistrias el primer Gobernante del naciente Estado Griego.

Palabras clave: Ilustración griega - Koraís - Revolución griega.

\section{ADAMANDIOS KORAÍS, LAST REPRESENTATIVE OF THE GREEK ILLUSTRATION}

Abstract: Among the great figures of the Greek Enlightenment, we can distinguish that of Adamandios Korais. Born and trained initially in Smyrna, he studied medicine in Montpellier, while entering classical philosophy. He settled in Paris in 1788, where he lives until his death. He develops an intense pedagogical activity and as editor of patriotic and pedagogical political texts and a vast Greek Classical Library. While he strongly supported the Revolution of the Independence of Greece (1821-1830), he criticized the divisions among the patriots. He also criticized the work of Ioanis Kapodistrias the first Ruler of the nascent Greek State.

Keywords: Greek Enlightenment - Korais - Greek Revolution.

Recibido: 10.11.2018 - Aceptado 15.01.2019

Correspondencia: Miguel Castillo Didier

Email: micastilgriego@gmail.com

Director del Centro de Estudios Griegos, Bizantinos y Neohelénicos

Profesor Titular de la Universidad de Chile 
Este trabajo forma parte de un proyecto de investigación, registrado en la Facultad de Filosofía y Humanidades 2017-2018, sobre Eugenio Vúlgaris y la Ilustración griega.

orkheimer y Adorno definen la Ilustración como un proceso
de larga data: "La Ilustración, en el más amplio sentido de
pensamiento en continuo progreso, ha perseguido desde siempre el objetivo de liberar a los hombres del miedo y constituirlos en señores [...]. El programa de la Ilustración era el desencantamiento del mundo. Pretendía disolver los mitos y derrocar la imaginación mediante la ciencia". Esta última afirmación puede aplicarse más concretamente al proceso que se vivía en Europa desde fines del siglo XVII.

Como escribe Pasjalis Kitromilidis, "La filosofía de la Ilustración expresó la emancipación del pensamiento europeo de las ataduras de la cosmovisión medieval. Al enterrar los presupuestos filosóficos del pensamiento medieval y al ofrecer un conjunto de alternativas de principios cognoscitivos y valóricos, la filosofía de la Ilustración reivindicaba el predominio del espíritu contemporáneo, después de las largas luchas espirituales y políticas que habían dado forma a la historia cultural de Europa desde el Renacimiento en adelante"2. La Ilustración trataba de liberar el pensamiento de la ignorancia, del prejuicio, de engaños seculares. Sin duda, sus fuentes eran "la radical duda cartesiana frente a las autoridades consagradas y la lucha implacable que habían proclamado Hobbes y Spinoza contra la superstición que humillaba al espíritu humano"3. La reflexión en torno a la naturaleza del conocimiento, sus fuentes y sus límites, que había planteado John Locke en su obra Ensayo sobre el entendimiento humano, en 1689, determinando así las potencialidades cognitivas del hombre, puede considerarse el punto de partida del gran proceso de la Ilustración. Un siglo después, en 1784, otro pensador, Emanuel Kant, podía definir así tal proceso:

1 Korkheimer, M. y Adorno, Th. (1998), p. 59.

2 Kitromilidis, P. (2009) Neohelinikós Diafotismós I politikés ke kinonikés idees Ilustración griega Las ideas políticas y sociales, p. 13.

3 Ibídem, loc. cit. 
"La Ilustración es la liberación del hombre de su culpable incapacidad. La incapacidad significa la imposibilidad de servirse de su inteligencia sin la guía de otro. Esta incapacidad es culpable porque su causa no reside en la falta de inteligencia sino de decisión y valor para servirse por sí mismo de ella sin la tutela de otro. ¡Sapere aude! ¡Ten el valor de servirte de tu propia razón!: he aquí el lema de la Ilustración"4.

Las palabras de Kant que definían la Ilustración a finales del siglo XVIII se referían al movimiento conocido por el nombre de Aufklärung en alemán, Les Lumières en francés, Illuminismo en italiano, Enlightenment en inglés, Ilustración en castellano. Posiblemente no podía saber Kant que un movimiento semejante, pero de ciertas connotaciones distintas y un tanto atrasado en el tiempo, se estaba produciendo en la región del sudeste europeo, dominado desde hacía siglos por el Imperio Otomano, y que llegaría a denominarse, muy tardíamente, con el neologismo griego

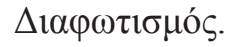

$\mathrm{Y}$, en realidad, la Ilustración griega se produce en regiones que estaban desde hacía siglos bajo el duro dominio otomano. La renovación del pensamiento proviene en parte de los pocos griegos que podían hacer estudios en Occidente, especialmente en Italia, y que regresan al territorio subyugado; en parte de los griegos que viven en los Principados Danubianos que gozan de autonomía, bajo soberanía otomana; en parte de las traducciones de obras de filósofos y científicos occidentales, realizadas por estudiosos con gran vocación pedagógica, como Eugenio Vúlgaris (1716-1806), Iósipos Misiódax (1830-1780), Demetrio Katartzís (c. 17301807), Rigas Velestinlís (1757-1798). Y en éstos y demás representantes de la Ilustración griega, que viven y actúan en tierras helénicas y rumanas, se une la ideología de la Ilustración con el anhelo de liberación de la secular tiranía otomana. No pocos de los ilustrados griegos debieron vivir parte importante de sus existencias fuera de Grecia y las obras que escribieron y tradujeron se publicaron en Venecia, Bolonia, Leipzig, Viena, París, Moscú, San Petersburgo. Sólo excepcionalmente algunos escritos de ilustrados vieron la luz en Constantinopla o Ioanina.

4 Kant, E. (1994) Filosofía de la Historia, p. 25. 
Tratamos de presentar la figura de Vúlgaris y de no pocos de los grandes representantes de la Ilustración griega en un amplio trabajo de investigación, titulado Eugenio Vúlgaris y la Ilustración griega.

Aquí, presentamos la personalidad y obra de Adamandios Koraís (1748-1833), quien, proveniente del Asia Menor, pasó gran parte de su vida en Francia.

\section{Nacimiento en Esmirna y primeros estudios}

Koraís nació en Esmirna en una familia de comerciantes cultos. Estudió en la prestigiosa Escuela Evangélica de su ciudad natal, institución que mantenía una severa disciplina. Desde muy temprana edad mostró interés en adquirir nuevos conocimientos. A los obtenidos en sus estudios regulares, añadió el de lenguas extranjeras bajo la enseñanza de un sacerdote holandés, Bernhard Keun, que vivía en Esmirna. Adamandios, a su vez, enseñó griego a su maestro.

$\mathrm{Su}$ padre, comerciante en sedas, lo envió en 1771 a Holanda a dirigir la sucursal de sus negocios en la ciudad de Ámsterdam, donde podría también tener la oportunidad de ampliar su cultura. Recomendado por su maestro Keun, fue acogido por el profesor Andrien Buurt, con quien estudió los Elementos de Euclides y lógica. Rápidamente fue integrándose a la vida cultural de la ciudad, estudiando música y teatro y siguiendo cursos en la Universidad Libre Ateneo.

Permaneció seis años en Ámsterdam. En 1777, la familia decidió que regresara a Esmirna. En el viaje de retorno se detuvo en Viena y en Venecia, mientras trataba de convencer a sus padres que le permitieran estudiar medicina en Montpellier. Pero no obtuvo permiso y volvió a Esmirna en junio de 1778. Cuatro años permaneció en la ciudad y, como consecuencia de problemas de salud y de ánimo, por fin, en 1782, le fue permitido le fue permitido viajar a Francia para ingresar a la célebre Escuela de Medicina de Montpellier.

\section{La medicina y la filología clásica}

Junto con realizar sus estudios de medicina, Koraís se aplicó intensamente al estudio de los clásicos griegos y latinos y de los filósofos modernos occidentales. Se iniciará así en la filología clásica, disciplina en la que se destacará con brillo.

En 1783 y 1784 perdió a sus padres, lo que le trajo problemas económicos, que logró superar haciendo traducciones al francés desde el 
alemán y el inglés; además, recibió ayuda de su ex maestro Keun y de algunos amigos.

En 1786 publica su trabajo de título Sinopsis de Piretología y al año siguiente sus tesis de doctoral Un médico hipocrático. Entre tanto se lo ha integrado a la docencia en la Universidad de Montepellier, en la cátedra de "El corazón, las arterias y las venas".

Dos años después, en 1788, deja sus éxitos académicos y decide partir a París, para dedicarse por entero a las letras y a la causa nacional griega.

En una carta explica su decisión: “Abandoné el comercio, después de haberlo ejercido ya por seis años, porque me llevó a un círculo de muchas relaciones y dependencias molestísimas para mi indómita alma. Abracé la calidad de hombre de letras como la única dentro de la cual podría conservar mi independencia" ${ }^{5}$.

\section{En París hasta su muerte}

Es así como ese año llega a la capital francesa, donde permanecerá hasta su muerte. Vivirá, por lo tanto, todo el proceso de la Revolución Francesa, el Imperio, la Restauración Borbónica y los comienzos del reinado de Luis Felipe de Orleans. Y, desde París, desde lejos, vivirá todo el período prerrevolucionario de Grecia y toda la Guerra de la Independencia, incluida la imposición por las potencias europeas de una monarquía alemana al nuevo Estado.

El año 1789 vive en París el estallido de la Revolución, lo que le causa gran entusiasmo. Traduce enseguida al griego la Proclamación de los derechos del hombre y del ciudadano. Pero los excesos y los crímenes del Terror le merecieron las más duras condenas. También tuvo críticas para Napoleón y no aceptó el ofrecimiento que le hizo éste del cargo de Director de la Censura, por ser ésta incompatible con sus convicciones libertarias.

Sigue entre tanto haciendo traducciones de libros de medicina al francés para subsistir, pero pronto se centrará en una acción editorial de largo aliento, con la cual quiere contribuir a la liberación de su pueblo. Pero ya en 1798 un folleto con el título de Enseñanza fraterna, escrito con motivo

5 Citado por Anyelu, A. (1983) "Estilo y carácter de Koraís. Una primera aproximación al tema", p. 203. 
de la muerte de Rigas Velestinlís y sus siete compañeros ${ }^{6}$, entregados por el cristiano emperador de Austria Francisco a los otomanos, a sabiendas de que sufrirían horribles torturas y que finalmente serían asesinados. El escrito es una respuesta al folleto Enseñanza paterna, publicado por el patriarca de Jerusalén Ánthimos, con el pseudónimo de Atanasio Parios. En él, el patriarca defendía abiertamente el dominio otomano y llamaba a los griegos a no desear la libertad, a la vez que a rechazar la Ilustración.

Son emocionantes las palabras con que Koraís recuerda a los mártires. Escribe que al saber la noticia del martirio de los ocho patriotas, sintió que dentro de él" la Hélade toda, con lágrimas en los ojos" le dictaba estas palabras:

"Recientemente, el emperador de Alemania, aunque atormentado por una dolorosa y mortal enfermedad, que debiera siquiera enseñarle el amor y la simpatía al ser humano, entregó inmisericordemente al tirano de la Hélade a ocho griegos; ocho griegos que buscaban pacíficamente los medios adecuados para ilustrar a sus compatriotas y liberarlos del yugo de la esclavitud, han sido martirizados en la flor de la edad la noche del 24 de junio de 1798. Se vierte la generosa sangre griega de sus venas y vuelan sus almas benditas a reunirse con las inolvidables almas de los que han muerto por la Libertad [...]. ¡Este derramamiento de sangre inocente en vez de atemorizar a los griegos más bien los moverá a venganza!"’

El cómo llega Koraís a ser un ilustrado y participar activamente en las actividades de los ilustrados ha sido objeto de dos tesis. La de Dimarás, consignada en su obra Ilustración griega, $4^{\text {a }}$ edición 1985 , y la de Panayotis Kondilakis, expuesta en La Ilustración griega Las

6 Véase la sección dedicada a "Rigas y la Ilustración revolucionaria”, en Vúlgaris y la Ilustración griega (2018), donde se encontrará bibliografía sobre el tema.

7 Koraís, A. (1798, 1983) Adelfiki didaskalía, París 1798, Reedición facsimilar, pp. IV-V. En la primera edición, después de la frase "ocho griegos que...", Koraís escribió "Se presentan a esta misma hora ante el tirano estos valerosos mártires de la libertad; quizás a esta misma hora baja sobre sus sagradas cabezas el puñal del verdugo. Se vierte la generosa sangre griega..." 
ideas filosóficas, $3^{\mathrm{a}}$ edición 2008. Para Dimarás, "la relación de Koraís con la Ilustración debe entenderse en el marco de su contacto personal y espiritual con una determinada corriente de la Ilustración, es decir, con el movimiento de los llamados "Ideólogos"" "Esto podría significar que Koraís fue iniciado en las ideas de la Ilustración por los Ideólogos, o que sigue a éstos en sus conceptos filosóficos, es decir, que toma su posición en sus debates gnoseoteoréticos u ontológicos con otras escuelas filosóficas. Pero no sucede ni lo uno ni lo otro". En apoyo de su tesis, Kondilakis recurre a antecedentes documentados con anterioridad a la llegada de Koraís a Francia y su toma de contacto con los Ideólogos. "Ya en la época en que Koraís podía ser considerado un 'cristiano ilustrado' más que un ilustrado propiamente tal, formula convicciones de las que no se alejará durante toda su vida, como por ejemplo que la virtud es acción y no dogma o que él mismo quiere mantenerse 'a igual distancia de la Escila de la incredulidad y de la Caribdis de la superstición' ${ }^{10}$. Es notable que en la misma época Koraís se exprese elogiosamente de Rousseau, lo que no significa naturalmente la acogida de las ideas roussonianas en detalle, pero atestigua irrefutablemente la temprana disposición favorable de Koraís frente a los máximos representantes de la Ilustración" " "Con esta disposición llega Koraís a Francia en mayo de 1788 y precisamente la preexistencia de tal disposición explica por qué, como muestran sus cartas, de inmediato se entusiasma tanto por la tensión que entonces había de las fermentaciones políticas y culturales. Y siente como si entrara a un mundo nuevo. Siente que allí puede darle realidad a su lema: "Quien tiene un alma indómita, piensa, habla y escribe bien".

El estallido de la Revolución lleva al máximo su impresión de que se encuentra en el centro de acontecimientos mundialmente históricos, los cuales, sin embargo, considera engendrados por la génesis y la difusión de determinadas grandes ideas. Desde el momento en que en su mente se asienta y se consolida la relación de causalidad entre la Revolución y la Ilustración, debe considerarse que su entusiasta saludo a la primera equivale automáticamente a una igualmente entusiasta confesión de fe en

8 Dimarás, K. Th. (1985) pp. 111, 338.

9 Kondilakis, P. (2008), pp. 201-202.

10 Carta de 12.11.1785, citada por Kondilakis P (2008), p. 203.

11 Carta de 11.8.1783, citada por Kondilakis , P. (2008), p. 2003. 
la segunda.

Más allá y por sobre cualquier influencia libresca, la Revolución Francesa - como hecho social y como hecho ideológico - es la que transforma a Koraís de 'cristiano ilustrado' en ilustrado propiamente tal, para el cual ya el cristianismo sólo puede justificarse en armonía con la razón soberana. Como punto de total y consciente paso a la Idea y a las ideas de la Ilustración debemos, con todo, considerar, la revelación, al seguir el traslado de los restos de Voltaire al Panteón, de su anhelo de ser él mismo un Voltaire griego"12. Desde el balcón de su casa ve pasar el cortejo que va desde las ruinas de la Bastilla, donde habían sido depositados la tarde anterior, al Panteón. Se emociona leyendo las inscripciones del carro fúnebre, una de las cuales dice "El hombre tiene el poder y el derecho a derribar a sus tiranos"; en otra que va en uno de los costados de la caja que sostiene la estatua yacente de Voltaire, sobre la urna que contiene sus restos se lee: "Poeta, filósofo, historiador, que iluminó el espíritu humano y nos preparó para recibir la libertad". Y muy especialmente le emociona el estante que ostenta, encuadernados en oro los setenta tomos de las Obras Completas. Le recuerda las obras de los grandes escritores griegos antiguos. Sigue finalmente el cortejo que demoró nueve horas desde la Bastilla a la iglesia, donde llegó a medianoche. Toda la escena es descrita con detalle en carta a su amigo, de su ciudad natal Esmirna, el primer cantor Demetrio Loto, carta fechada el 15 de noviembre de 1791. Hay que recordar que en 1778, cuando murió Voltaire, la Iglesia no permitió que se lo sepultara en París ${ }^{13}$.

$\mathrm{Su}$ oposición a las supersticiones que adormecen al hombre, su valoración de la recta razón, como guía de toda conducta humana; su amor por la libertad y su rechazo a toda tiranía, que lo hará condenar a Robespierre como tirano y al período de su dictadura como una "época de canibalismo y barbarie"; su permanente dedicación a escribir y a editar para "llevar luces" a su pueblo"; sus múltiples gestiones, cartas, exposiciones, peticiones para contribuir a la libertad de su patria oprimida, lo sitúan plenamente en el panorama de la Ilustración griega.

12 Carta de 15.11.1791, citada por Kondilakis., P. (2008), p 204.

13 La carta se reproduce en Fasulakis, S. (1987) "El traslado de los restos de Voltaire a París (1791)", pp. 24.25. 
La primera mención de un Ideólogo en los escritos de Koraís es la de Volney en julio de $1793^{14}$. Este autor es mencionado en relación con la preocupación nacional de Koraís. Se refiere a él como un escritor que expresa sentimientos antiturcos. En efecto, la obra de Volney Considérations, proponía el abandono del apoyo a los otomanos frente a Rusia, sosteniendo que la desmembración del Imperio turco no perjudicaría sino que favorecería los intereses occidentales y más aún los de Francia. Coincidía así con los planteamientos de Vúlgaris en sus Reflexiones sobre el estado crítico actual dela Potencia Otomana en 1772.

Así pues, puede afirmarse que Koraís es un ilustrado consciente antes de que aparecieran las obras de los Ideólogos o antes que se hicieran conocidos los nombres de quienes más tarde tomaron ese nombre. Su familiaridad con los clásicos ilustrados la comprueba Kondilakis con una revisión de las notas de los folletos primeros de Koraís y de sus primeras ediciones de textos clásicos. La mayor parte de las menciones de autores son a Montesquieu: el Esprit des Lois; a Voltaire: el Dictionnaire Philosophique, a Rousseau: el Contrat Social; a D'Alembert: Sur la destruction des Jésuites en France. Tampoco faltan menciones de Bayle, Batteux, Condorcet, Vattel, Shaftesnury, Bentham y A. Smith ${ }^{15}$.

En cuanto a su posición ante el despotismo ilustrado de Catalina de Rusia, su posición en una época es análoga a la de Vúlgaris. Menciona con entusiasmo a la Emperatriz, en parte quizás siguiendo el ejemplo de algunos enciclopedistas, pero acaso principalmente porque, al igual que Vúlgaris esperaba beneficios para los griegos de la política antiturca de Catalina.

\section{El ilustrado y la gran Biblioteca Helénica}

Su trabajo filológico, cuya motivación es esencialmente pedagógica y patriótica, comienza en 1799 con la publicación de Los caracteres de Teofrasto, en una edición bilingüe con estudio introductorio y con notas. En los Prolegómenos a los tomos que van formando una larga serie, la monumental Biblioteca Griega, Koraís expondrá ideas acerca de la liberación de los griegos y sobre la conducta que deben tener para obtenerla. Su objetivo es contribuir a levantar el nivel cultural del pueblo griego y, a la vez, estimular en ellos el anhelo de liberación.

14 Carta de 28.7.1793, citada por Kondilakis, P. (2008), p. 205.

15 Kondilakis, P. (2008), p. 206. 
El año 1800 publica el poema Canto Guerrero y al año siguiente Clarinada Guerrera ${ }^{16}$, que constituyen llamados a los griegos a luchar ahora cuando la expedición de Napoleón a Egipto despertaba esperanzas de que Francia apoyara la causa helénica. Luego publica una Exposición sobre la actual situación de Grecia, y enseguida, en 1805 el folleto ¿Qué deben hacer los griegos en las presentes circunstancias? En 1802 edita su traducción de Acerca de los delitos y las penas de Beccaria (la que se reeditará veinte años después), con el fin de que pudieran utilizarlos los futuros jueces griegos.

Entre tanto, continúa con sus trabajos sobre autores antiguos, los que pronto le dan fama de filólogo. En 1800, aparece Sobre los vientos, aguas y lugares de Hipócrates. Mientras prepara un estudio sobre Dafnis y Cloe de Longo, publica en 1804 Las Etiópicas de Heliodoro (1804); Eliano y otros (1805); Isócrates (1805), con un estudio; Polieno (1809). Busca y consigue ayuda económica de amigos comerciantes y es así entre 1805 y 1827, tomará forma sistemática la Biblioteca Griega. Muchos son los autores antiguos cuyos textos conforman esta Biblioteca: Homero, Aristóteles, Isócrates, Plutarco, Estrabón, Jerocles, Jenócrates, Polieno, Galeno, Marco Aurelio. Mencionaremos otros autores publicados en los años de la Revolución de la Independencia.

En 1810, el Instituto de Francia premió su obra sobre Hipócrates, lo que le permitió financiar una segunda edición en 1816.

En los "Prolegómenos" a sus ediciones clásicas expone sus ideas acerca de la cultura y la educación de sus compatriotas, de la necesidad de trasladar la idea europeas a las escuelas griegas. Propone el uso de una lengua semi popular que facilite el estudio de los niños y los jóvenes de su patria. Un poco más tarde, amigos del maestro financiarán la publicación de un tomo que concentrará sus "Introducciones".

Sus obras lo fueron haciendo conocido en el círculo de los hombres de la cultura, griegos y los franceses. Llegó a ser amigo de filólogos cono Sarton de la Posset y De Villoison. Se hizo miembro de la Societé des Observateurs de l'Homme, junto con diversos Ideólogos. En la Asamblea de la Sociedad de Observadores del Hombre leyó el 6 de enero de 1803 uno de sus escritos más importantes: "Mémoire sur l'état actuel de la

16 Con motivo de los 150 años de la muerte de Koraís, el Centro de Investigaciones Neohelénicas publicó una edición facsimilar de varias obras del Maestro, entre otras de la Clarinada Guerrera. 
civilisation dans la Grèce".

Toma contacto con los políticos franceses, holandeses y hasta norteamericanos, a los cuales se dirige intercediendo por la causa de los griegos, sometidos a un yugo secular. Escribe incesantemente, convencido de que Francia no perdería sino que ganaría dejando su apoyo al Imperio Otomano. Es decidor el hecho de que de 1300 cartas suyas ubicadas, hay 15 que no se dirigen a una persona determinada, sino a muchas. De esas 15 , 7 se refieren a la necesidad de crear escuelas, de conseguir maestros, libros, etc. Las restantes tratan temas políticos. La primera de ellas se dirige a los dirigentes del Peloponeso y expresa su inquietud ante las divisiones de los patriotas, en plena lucha contra el poderoso Imperio Otomano. Las disensiones entre los combatientes lo angustiarán durante toda la Revolución de la Independencia y en los pocos años que alcanzó a ver a Grecia independiente ${ }^{17}$.

Koraís, que había aceptado la ciudadanía francesa, rechazó ofrecimientos importantes que habrían podido regularizar su situación económica. En 1814 no aceptó la propuesta de trabajar en el Collège de France, después de la muerte de Bosquillon. Antes se había negado a ocupar el puesto de Villoison en la misma institución. Más tarde, en 1816, rechazó el ofrecimiento para que asumiera una cátedra en el Instituto de Francia. Ocupado intensamente en sus trabajos filológicos y patrióticos, prefirió desechar esas oportunidades.

\section{Koraís y la Revolución de 1821}

Al estallar la Revolución Griega, en 1821, Koraís, pese a que en esa época pensaba que tal hecho debía producirse hacia mediados de siglo, presta todo su apoyo a los patriotas. Publica un llamado a los griegos e inserta la Proclama del general Alejandro Ipsilandis quien inició la Revolución de la Independencia en Moldavia en febrero de 1821: Appel aux Grecs. Traduit du grec moderne d'Atromète, natif de Marathon, avec la Proclamation d'Ypsilandi aux Français. Es la traducción de la Clarinada Guerrera, con introducción y comentarios de Koraís. En carta a su amigo Vlastós, del 23 de mayo de 1821, escribe: "Siento una alegría tan grande que no puedes imaginártela”. Aprueba con énfasis el que los hermanos de Vlastós haya corrido "en auxilio de la patria" y agrega, a continuación: "Si yo tuviera veinte años menos ni dioses ni demonios me impedirían

17 Fasulakis, St. (1992), pp. 423-424. 
ir a la revolucionada Grecia" 18 . Sin embargo, en la misma carta, junto a su entusiasmo añade también sus dudas "Para derribar un gran edificio no se necesita sabiduría [...]. La grande y terrible dificultad está en la construcción, la que requiere arquitectos como Arístides y a otros como era Arístides, de los cuales no veo ninguno en la nación. En vez de esos, tenemos muchos mediosabios, muchos pretenciosos, los cuales desearían ser demagogos y ociosos en un pueblo todavía sin instrucción"19.

En diciembre de 1821, al recibir noticias de las primeras disensiones entre los patriotas, escribe a un amigo,: "La cosa comenzó antes de tiempo en un pueblo que no tiene todavía suficientes luces para comprender sus verdaderos intereses [...]. Si hubiera comenzado veinte años después, entonces se habrían hallado más maduros los frutos de Quíos y de Kidoniés, por una parte, y del mayor número de jóvenes que hubieran estudiado en Europa" ${ }^{20}$. Alude aquí Koraís a las dos importantes escuelas de estudios superiores de la isla de Quíos y de Kidoniés (Aivalí) en Asia Menor.

Quisiera editar, como un aporte, a todos los escritores antiguos que trataron de ética y de política, si sus medios económicos se lo permitieran. Pero al menos elige para continuar la Biblioteca Griega textos de Aristóteles, Platón, Jenofonte, Licurgo, Plutarco, Arriano y otros. Quisiera participar personalmente en el combate revolucionario: "Si tuviera veinte años menos de edad, ni dioses ni demonios me lo impedirían", escribe ${ }^{21}$, como lo vimos. A sus 73 años, sigue día a día los pormenores de la Revolución y luego ve con inquietud los primeros pasos del naciente pequeño Estado helénico.

Durante toda la época de la Revolución y desde mucho antes de su estallido, una preocupación central de Koraís es el problema de la educación en Grecia.

Redacta manuales que envía a las escuelas; propone métodos nuevos de enseñanza; propone el uso de una lengua "sencilla", aunque no propiamente la hablada, y no la antigua, a fin de facilitar la enseñanza; pide a sus compatriotas que traduzcan textos útiles para elevar el nivel cultural

18 Carta citada por Politis, A. (2007), p. 240.

19 Politis, A., ibídem, loc. cit.

20 Carta el 24 de diciembre de 1821 a Jacobo Rotas, citada por Poltitis, A. (2007), pp. 241-242.

21 Citado por Dimarás, K. Th. (1985), p. 110. 
del pueblo y para su manejo en la educación. Su preocupación pedagógica, más bien su pasión por ilustrar a su pueblo, nunca lo abandonó.

Y sin duda pensaba que una tarea fundamental del nuevo Estado griego debía ser la educación gratuita. En una carta a La Rochette, unas palabras retratan su posición al respecto: “¡Cuán grande fue mi pena cuando al llegar a París [en 1788], me vi obligado a comenzar a enseñar griego por dinero! El día que recibí mi primer pago fue para mí un día de duelo. Me sucedió lo mismo que Plutarco cuenta de Isócrates"22.

Desde Francia ve que el Estado libre y democrático que soñaba está cayendo en lo que considera una dictadura, bajo el régimen del primer Gobernante, Ioanis Kapodistrias, con quien había mantenido amistosa correspondencia. Y escribe criticando duramente al Gobernante, dos diálogos con el mismo título de ¿Qué conviene en las actuales circunstancias a la Grecia liberada de los turcos para no ser esclavizada por cristianos turquizantes? Con amargura debe haber reaccionado Kapodistrias ante esa crítica. Y Koraís publica en octubre de 1831, ignorando el asesinato ${ }^{23}$ del Gobernante, otro folleto titulado Miscelánea Griega desde el comienzo del Gobierno de Kapodistrias en adelante, igualmente crítico. La segunda parte de este texto apareció en agosto de 1832.

Hay que reconocer que Koraís no podía imaginar desde Francia el verdadero estado de cosas que había en Grecia, un país devastado por la guerra, en la ruina económica, carcomido por las divisiones internas, los caudillismos, las ambiciones y las pequeñeces políticas. La misma Asamblea Nacional de Trezena, de 1827, de espíritu realmente democrático, que había enviado una hermosa carta de reconocimiento a Koraís, que había elegido a Kapodistrias como primer Gobernante, y, que, a petición de éste, le había otorgado poderes especiales, tuvo conciencia del estado anárquico en que se encontraba el país. No había otra posibilidad de intentar salvar la situación y consolidar el Estado naciente que el camino que proponía y trató de seguir Kapodistrias ${ }^{24}$. Todo esto no lo vivía Koraís

22 Koraís, A. (1983) “Escrito autobiográfico”, p. 11.

23 Kapodistrias cayó asesinado en Nauplio, primera capital del Estado griego, el 27 de septiembre de 1831. Sus últimas palabras fueron "Concordia, hermanos".

24 Los esfuerzos del Gobernante por poner orden y organizar un país devastado y anarquizado y el choque con el odio de caudillos opositores son materia de una impresionante tragedia de Nikos Kazantzakis, titulada Kapodistrias. Traducida $\mathrm{y}$ con un estudio introductorio la hemos presentado en el volumen Nikos 
ni podía imaginarlo. Además, es posible que los enemigos del Gobernante en Francia le presentaran la situación como ellos querían verla. También pueden haber influido en él, a través de la correspondencia, los opositores internos que actuaban en Grecia.

Adamandios Koraís, el último de los más grandes representantes de la Ilustración griega, es el único que pudo ver, aunque de lejos, la Revolución y el nacimiento del pequeño Estado neogriego. Pero, también desde lejos, pudo saber, asimismo, de las divisiones, las ambiciones, los caudillismos, que llevaron a la naciente Grecia a la anarquía. Por eso, escribe en 1831. "La Revolución de Grecia era justísima, pero se hizo a destiempo. Su tiempo era el año 1850 cuando tendríamos a muchos de nuestros jóvenes que hoy estudian de edad de 30 y 40 años y otros de más de 40, enseñados por los acontecimientos ya ocurridos y los que ahora ocurren en Europa, capaces de manejar los asuntos y de disolver las facciones" 25 .

Tuvo la pesadumbre de alcanzar a saber de la imposición a Grecia por parte de las potencias de una monarquía absoluta, con un rey extranjero. Lejos estaba ese régimen del Estado democrático y liberal que él había soñado. El mismo año 1833, en que muere Koraís llega a Grecia el rey Otón, procedente de Baviera.

Otros Ilustrados vivieron el estallido de la Revolución, como Benjamín de Lesbos (+1824); Dimitrios Filipidis (+1832), Atanasio Psalidas (+1829). Constantino Kumas vio (desde Trieste) el régimen "bávaro" en pleno funcionamiento $(+1836)$. Constantino Ikonomos (1857) alcanzó a vivir, en Atenas, la Revolución de 1843, que impuso una Constitución a la monarquía.

Sus últimos años deben haber sido bastante tristes. En una carta escribió: "Hasta ahora he pasado una vida solitaria, temerosa y casi salvaje", "monotone et mélancolique"26.

Koraís murió, anciano y enfermo, a los 85 años, el 6 de abril de 1833, después de haber vivido 45 años en Francia. Su vida no fue fácil ni

Kazantzakis Teatro, Centro de Estudios Bizantinos y Neohelénicos, Santiago 1978.

25 Carta a Alejandro Kondóstavlos del 28 de enero de 1831. Citada por Politis, A. (2009), pp. 244-245.

26 Citada por Anyelou, Al. (1983) "Estilo y carácter de Koraís. Una primera aproximación al tema", pág. 205.

194 
alegre. Sí fue muy apasionada.

Había sido testigo de la Revolución, del Imperio de Napoleón, de la Restauración y de los primeros años del reinado de Luis Felipe de Orleans.

Dejó diversos trabajos no publicados o no terminados, como su Autobiografía, una Gramática de la Lengua Griega y un Diccionario francés-griego. Había escrito hasta el mismo año de su muerte. Como testimonio de su constante lucha - hecha a través de las letras y de la palabra - quedó una inmensa cantidad de cartas.

Como Vúlgaris, Koraís escribió hasta el final de su vida, muy poco más corta que la de aquél. Luchó, como el sabio de Kérkira, pero fue más crítico y más apasionado que éste. Polemizó con la Iglesia Ortodoxa y la criticó acremente, diferenciándose en esto de Vúlgaris cuya crítica a clérigos y a autoridades eclesiásticas fue más moderada. Pero, como escribía Pablo Kaligás en 1851, justificaremos la extraordinaria actividad de ambos y confesaremos que fueron Maestros de la Nación, cada uno a su manera" 27 .

\section{Referencias bibliográficas}

ANYELU, A. (1983). «"Y $\theta \varepsilon \dot{\mu} \mu \alpha\rangle$ "Estilo y carácter en Koraís Una primera aproximación al tema". Jornadas Korais: Atenas.

CASTILLO DIDIER, M. (2018). Eugenio Vúlgaris y la Ilustración griega. Centro de Estudios Griegos, Bizantinos y Neohelénicos: Santiago.

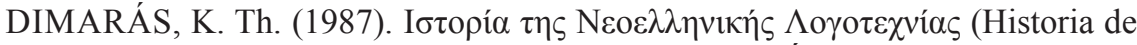
la Literatura Neohelénica). $8^{\mathrm{a}}$ edición. Editorial Íkaros: Atenas.

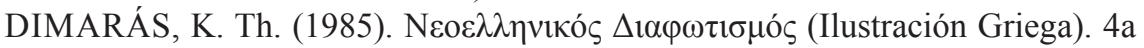
edición. Ediciones Hermís: Atenas.

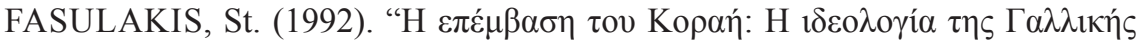

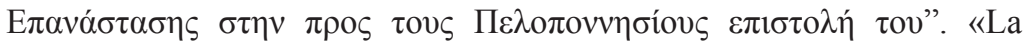
intervención de Koraís: La ideología de la Revolución Francesa en su carta dirigida a los peloponesios". En Anuario Cientifico de la Facultad de Filosofia de la Universidad de Atenas, vol. XXIX.

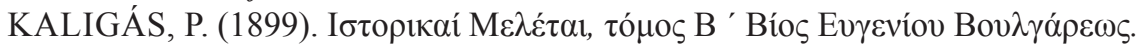
Estudios Históricos, tomo II Vida de Eugenio Vúlgaris. Nombre de la editorial ilegible: Atenas.

27 Kaligás, P. (1851), p. 241. 
KANT, E. (1994). Filosofía de la Historia. Traducción Eugenio Imaz. 5 reimpresión. FCE: México.

KITROMILIDIS, P. (2009). Neohelinikós Diafotismós I politikés ke kinonikés idees. Ilustración griega Las ideas políticas y sociales. Fundación Cultural del Banco Nacional de Grecia: Atenas.

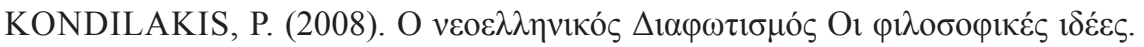
La Ilustración griega Las ideas filosóficas. Ediciones Themelio: Atenas.

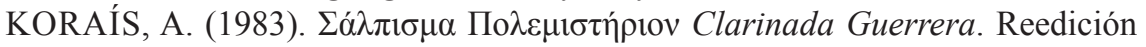
facsimilar del Centro de Investigaciones Neohelénicas: Atenas.

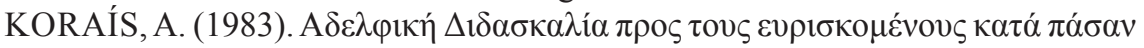

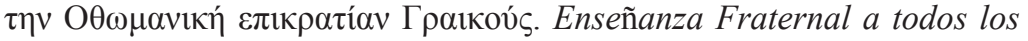
griegos que se encuentran en territorio otomano. Reedición facsimilar del Archivo Griego Literario e Histórico. Ediciones del ELIA: Atenas.

KORHEIMER, M. y ADORNO, Th. (1998). Dialéctica de la Ilustración. Fragmentos filosóficos. Introducción y traducción J. J. Sánchez. $3^{\text {a }}$ edición. Editorial Trotta: Madrid.

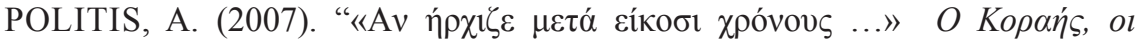

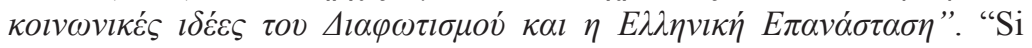
hubiera comenzado veinte años después... Koraís, las ideas sociales de la Ilustración y la Revolución Griega". Eranistís 26. 